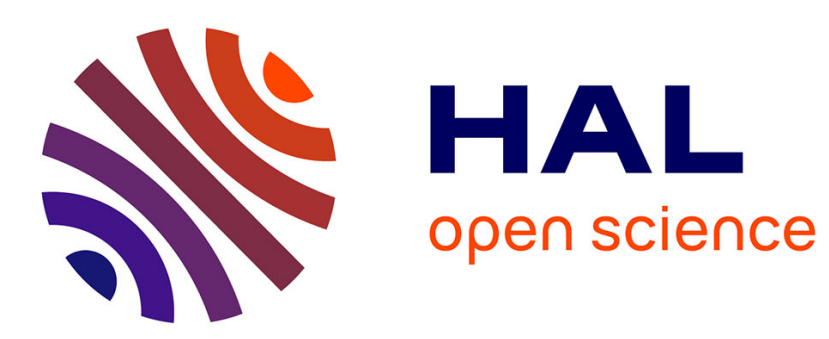

\title{
The Oscillatory 2D Convective States of a Binary Fluid Confined in Small Cavity
}

\author{
E. Delouche, G. Labrosse, E. Tric
}

\section{To cite this version:}

E. Delouche, G. Labrosse, E. Tric. The Oscillatory 2D Convective States of a Binary Fluid Confined in Small Cavity. Journal de Physique III, 1996, 6 (11), pp.1527-1534. 10.1051/jp3:1996200 . jpa00249543

\section{HAL Id: jpa-00249543 https://hal.science/jpa-00249543}

Submitted on 1 Jan 1996

HAL is a multi-disciplinary open access archive for the deposit and dissemination of scientific research documents, whether they are published or not. The documents may come from teaching and research institutions in France or abroad, or from public or private research centers.
L'archive ouverte pluridisciplinaire HAL, est destinée au dépôt et à la diffusion de documents scientifiques de niveau recherche, publiés ou non, émanant des établissements d'enseignement et de recherche français ou étrangers, des laboratoires publics ou privés. 


\title{
The Oscillatory 2D Convective States of a Binary Fluid Confined in Small Cavity
}

\author{
E. Delouche $\left(^{*}\right)$, G. Labrosse and E. Tric \\ CNRS - LIMSI, Université Paris-Sud, 91405 Orsay Cedex, France
}

(Received 7 May 1996, revised 1 July 1996, accepted 19 September 1996)

PACS.47.20.Bp - Buoyancy-driven instability

PACS.47.27.Te - Convection and heat transfer

PACS.47.20.-k - Hydrodynamic stability

\begin{abstract}
D accurate numerical experiments have been performed with a Boussinesq binary fluid subject to Soret effect and confined in a small (length/height $=2$ ) cavity. The steady convective states observed close to the threshold from conduction to convection, for a separation ratio fixed at -0.2 , are not made of standing waves. Rather, they can be seen as made of unbalanced and phase shifted counterpropagating traveling waves. The salient features of these flows are reported, their temporal characteristics in particular.
\end{abstract}

\section{Introduction}

Owing to the coupling they offer between buoyancy and distinct molecular diffusion characteristic times, the binary fluids with Soret effect [9] constitute a simple laboratory of interesting temporal behaviors when the concentration distribution of the basic (conduction) state acts to stabilize a fluid layer submitted to a destabilizing thermal vertical gradient. This configuration has been thoroughly explored and analysed, in the vicinity of the first (Hopf) bifurcation, with 2D horizontally infinite or extended cavities (see, for example, [1] and the references quoted therein). A large variety of oscillatory states is observed, essentially made of traveling waves, even in closed (but extended) cavities. Following the discussion presented in [4], standing waves (oscillations about the quiescent state!) are, "at first sight, the natural solutions in finite geometry". So, should these waves be effectively preferred, in sufficiently small cavities, to the traveling ones? Or, rather, should the steady convective flows be the only ones stable, as happens in the "phase pinning boundary condition" case of [1]?

\section{Modelisation}

Numerical experiments have been performed with a Boussinesq binary mixture confined in a small (radius/height $=2$ ) cylindrical cavity, and assumed to undergo axisymmetric $(r, z)$ convective motions. The momentum, energy and concentration equations, formulated as in [7],

(*) Author for correspondence (e-mail: delouche@limsi.fr) 
have been solved, using a monodomain pseudospectral Chebyshev method for the space derivatives evaluation [3] and a temporal second order finite differences scheme, the diffusion and convection terms being respectively implicitly and explicitly treated. The uncoupling between the velocity and pressure fields operators has been performed by a "projection - diffusion" method recently proposed in [2]. The imposed boundary conditions are (a) no-slip on the rigid walls $r=2$ and $z= \pm \frac{1}{2}$, (b) thermal adiabaticity on the lateral boundary and imposed temperature on top and bottom, $T\left(z= \pm \frac{1}{2}\right)=\mp \frac{1}{2}$, and (c) no mass flux through the walls.

Times are scaled with the vertical thermal diffusion time. The thermal Rayleigh number is the free parameter of this study, the Prandtl and Lewis numbers being fixed at 1 and 0.1 respectively, and the separation ratio taken equal to -0.2 .

Convergence to steadiness is declared when the following criterion is satisfied:

$$
\frac{\left|\phi_{n}-\phi_{n-1}\right|}{\left|\phi_{n}\right| \delta t} \leq 10^{-8} \text { for all } \phi
$$

where $\phi_{n}$ stands for the maximum absolute value, found on the nodes at time $n \delta t$, of one of the physical fields (the velocity components, the temperature and concentration). Time steps of $8 \times 10^{-4}$ have been used, corresponding to $\frac{1}{1000}$, or so, of the typical dimensionless main period of the observed oscillatory convective states. The chosen typical nodes number is $80 \times 30$ in $r \times z$ except for the $\mathrm{Ra}=2600$ which has been experimented with $220 \times 50$ nodes.

\section{Results}

Figure 1 gives the bifurcation diagram of the final non linear steady states. Identified by the right crosses are the oscillatory flows obtained starting from a randomly (to $10^{-2}$ ) perturbed conductive state. Both the rhomb states (the conductive and the stationary convective ones) have been obtained from the perturbed conductive states and, also, from the closest oscillatory one. As already known [11], the steady oscillatory states settle themselves with a lower frequency than the linear onset frequency, through a transient stage (given inserted in Fig. 1) which looks much like the one presented in [6]. We have here $\omega_{\text {hopf }}=9.1 \pm 0.1$. The frequency decreases as the $\mathrm{Ra}$ value increases, and exhibits an abrupt transition to the stationary state, occurring within the range $\mathrm{Ra} \in] 2875,2880]$. This is a salient feature of the flows in closed small cavities compared to what happens in the extended (or infinite, [1] for instance) cases where this transition is smooth. The lowest frequency solution ( $\mathrm{Ra}=2875$ ) has been run on a duration of 1700 units of time while the next one became suddenly stationary after a duration of 55 units of time (see the time trace inserted in Fig. 1). The onset to oscillatory convection occurs at $\mathrm{Ra} \in] 2550,2600]$, while $\mathrm{Ra}=2310$ is the Hopf threshold for our binary mixture in a horizontally infinite cavity [8]. In contrast with the results reported in [6], all the oscillatory states have their amplitude slightly modulated, at most to $10^{-3}$ in relative value, on the duration of our experiments, by a low frequency. This is illustrated in Figure 2 which gives an enlarged portion of the time trace signal envelop for the $\mathrm{Ra}=2600$ solution. It is of course very difficult to ascertain the steadiness of this modulation. Its frequency is well established and depends on the $\mathrm{Ra}$ value $(0.103 \pm 0.002,0.8 \pm 0.1$ and $0.40 \pm 0.02$ at $\mathrm{Ra}=2600,2700$ and 2875 respectively) while its amplitude might have not yet reached its final level. Thus, in the long duration experiment made with the $\mathrm{Ra}=2875$ flow, a $10^{-1}$ relative drift is still present. At last, the branch of stationary solutions has been explored on both sides of the $\mathrm{Ra}=2880$ stationary state, by starting from one of them.

From now on, we concentrate on the $\mathrm{Ra}=2600$ state to characterize its leading temporal behavior. Figure 3 gives instantaneous contour plots, in the cavity, at moments chosen in a half period, of the stream function, and of the departure from the conductive temperature $\theta$ 


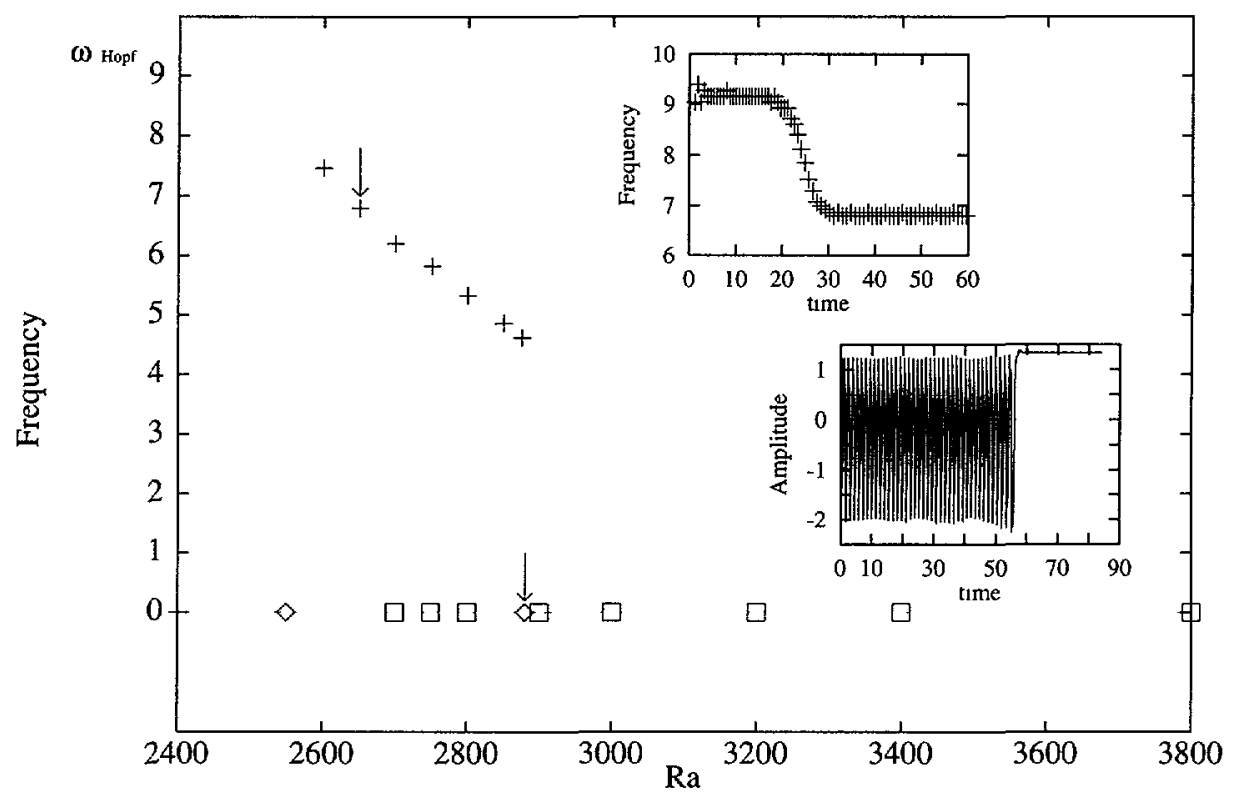

Fig. 1. - Observable regimes for $\psi=-0.2$ as a function of the Ra number. The leading frequency characterizes the oscillatory flows. The cross indicates states obtained starting from conductive state randomly perturbed. The rhomb states can be obtained by a similar way, or, starting from the nearest oscillatory case. The square states are obtained starting from one of the convective steady state. Inserted at the top, the time history of the leading frequency for Ra $=2650$ and, below, a time history of a component of the velocity for $\mathrm{Ra}=2880$.

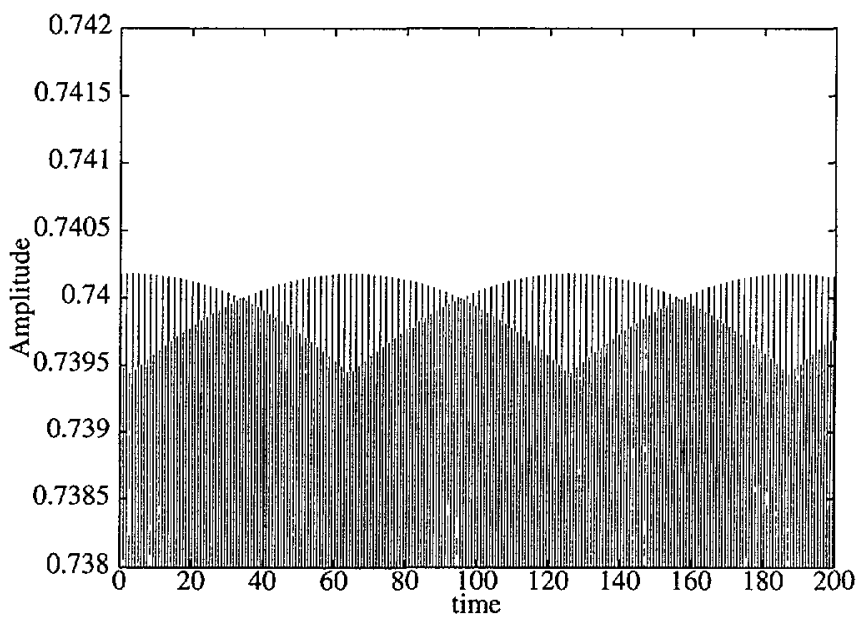

Fig. 2. - Time history for $\mathrm{Ra}=2600$ : enlarged portion of the signal envelop.

and concentration fields $\gamma$. The actual flow behavior does not emerge from such plots and an effective fluid particle path has been reconstructed on a duration of 300 periods and is presented in Figure 4. Each roll, thermally driven, acts like an oscillating sling and ejects fluid particles captured by the other roll, and permanent convective swaps take place between the rolls, inducing their small displacements and deformations. 

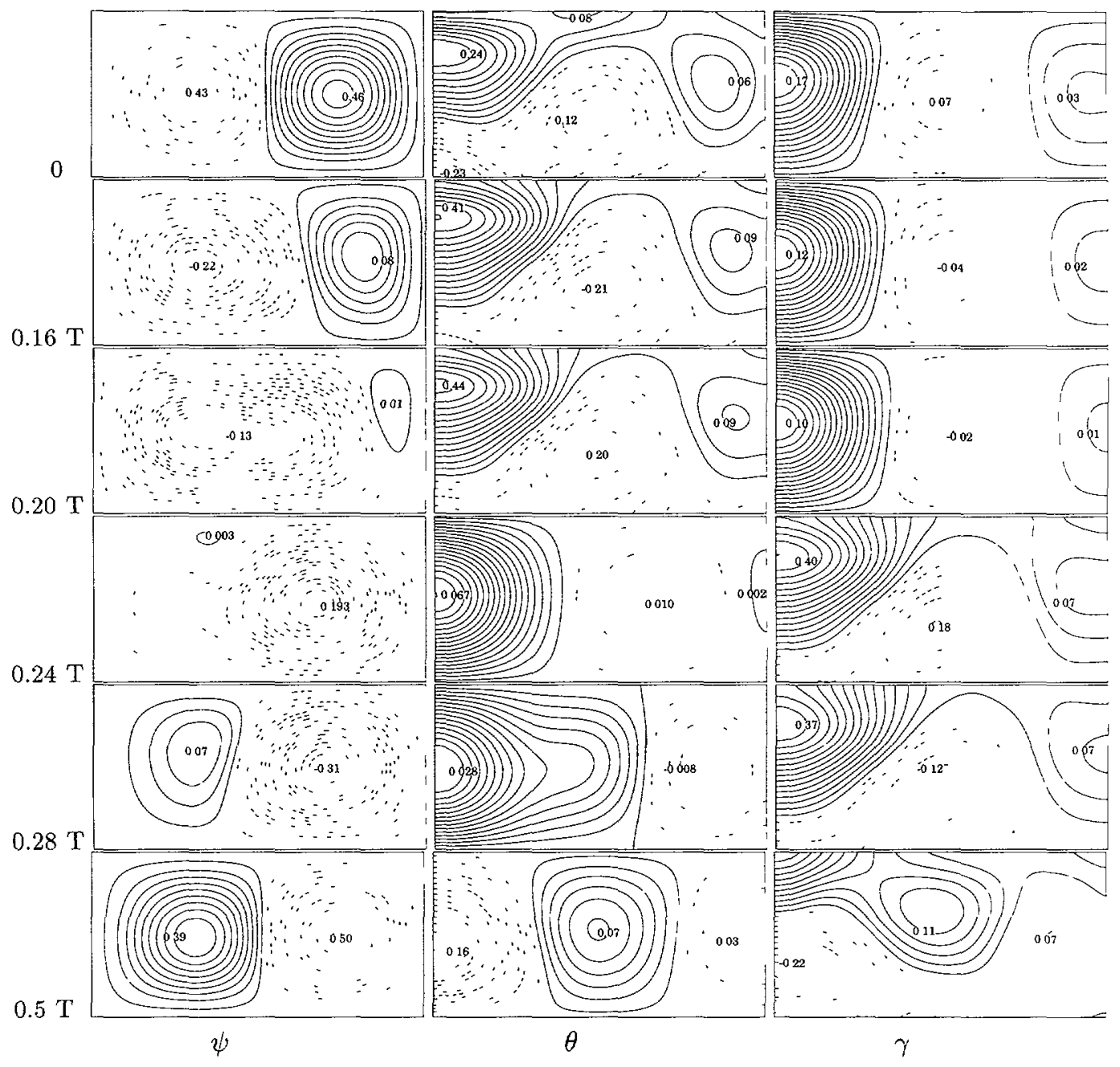

Fig. 3. - Flow visualisation for $\mathrm{Ra}=2600$ during $\frac{1}{2}$ period: stream function $\psi$, departure from the conductive state respectively from temperature, $\theta$, and concentration, $\gamma$; dashed lines indicate negative values.

This state is predominantly monoperiodic if one forgets about the above mentioned very low frequency $\left(\frac{1}{75}\right.$ here) modulation. In order to identify its temporal properties, we go now to present its time spectrum, computed in the cavity, for each of the velocity components and the temperature and concentration fields. The two vertical parity configurations have been taken separately, corresponding to the "even" flow expected to be triggered at the Hopf transition and to the "odd" one induced by the nonlinearities. Figure 5 gives the contour plots, for each parity case, of the modulus of the first temporal mode (whose frequency is plotted in Fig. 1). The "even" contribution (first column) is clearly one order of magnitude dominant over the "odd" one and is the only one considered in the sequel. 


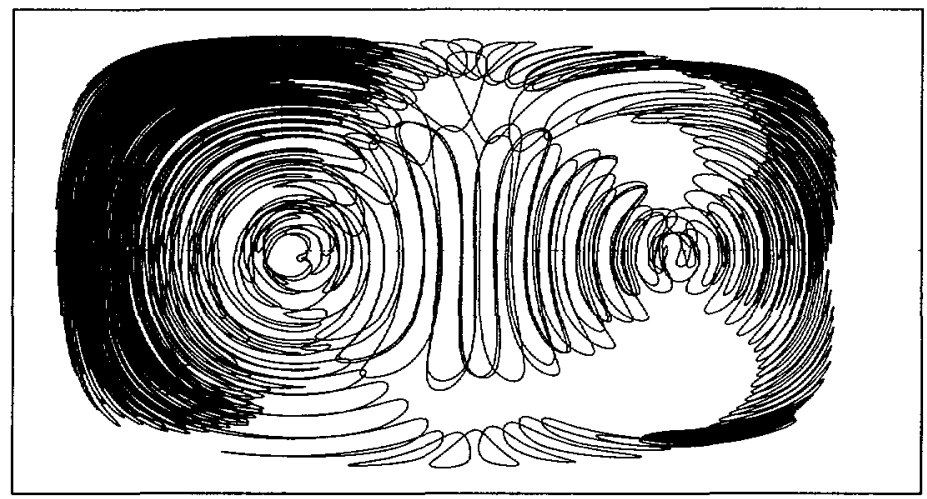

Fig. 4. - A fluid particle path in the cavity (from $r=1.5, z=0$ ) on a duration of 300 periods.
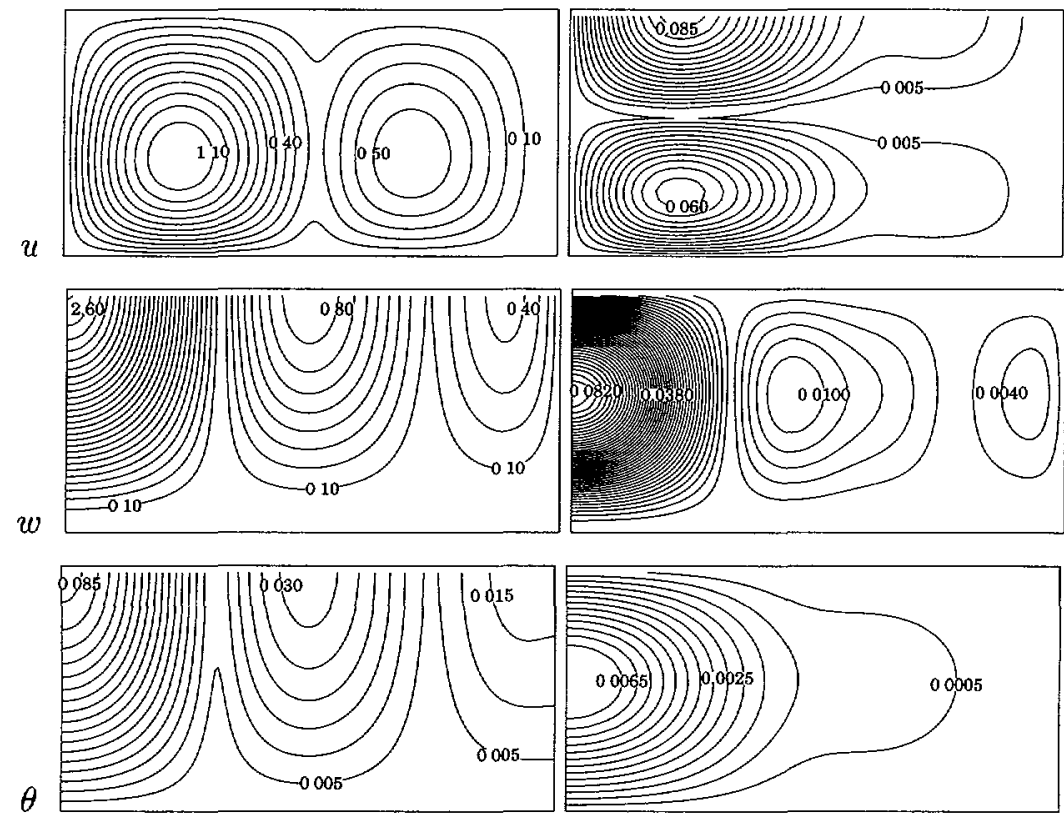

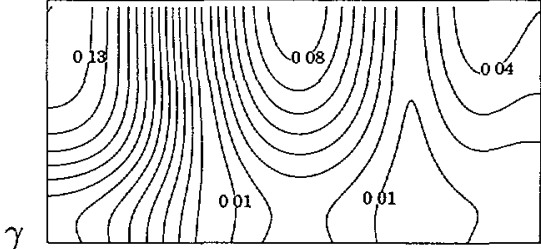

Even

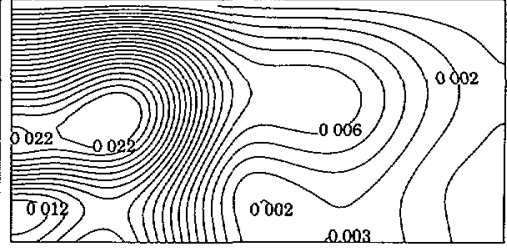

Odd

Fig. 5. - Contour plots for the "odd" and "even" contribution of the modulus of the first temporal mode; $u(w), \theta, \gamma$ are respectively radial (axial) component of the velocity, the departure from the conductive state for temperature and concentration. 
$w$

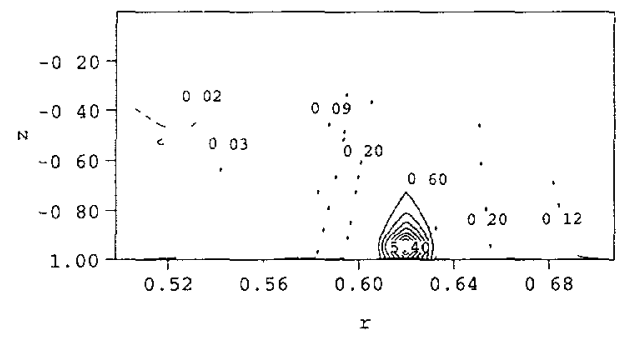

$\gamma$

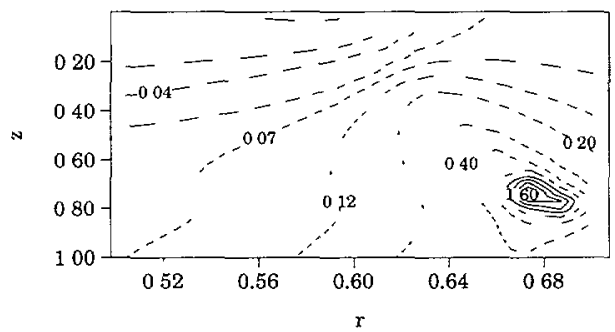

Even
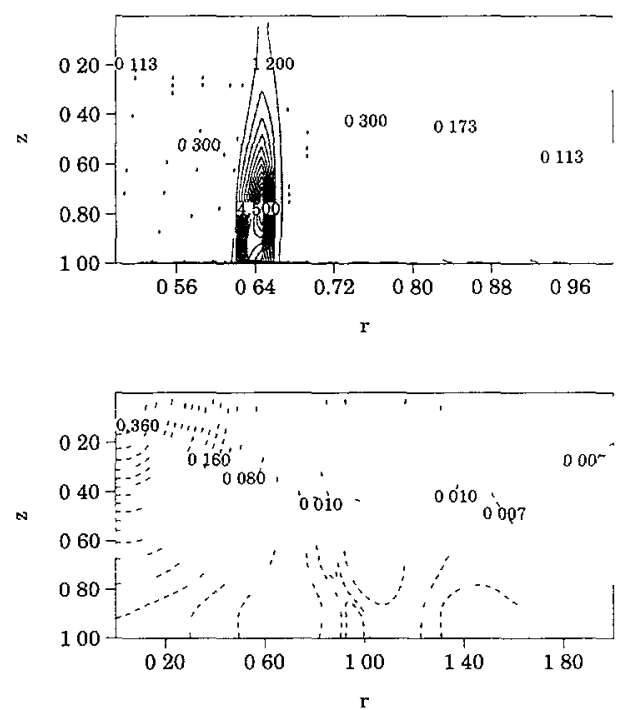

Odd

Fig. 6. - Contour plots of the ratio of the moduli: third mode/first mode.

Compared to the other harmonics, the first temporal mode is largely dominant everywhere in the cavity for the temperature and the velocity radial component, whereas, for the two other fields, it dominates almost everywhere except in a very small region where the third mode gets larger. Figure 6 presents the contour plots of the ratio of the moduli of the corresponding amplitudes for the velocity vertical component and the concentration fields, in the region of the cavity where the third mode dominates on the first one. This effect is quite significant for the axial velocity since the ratio increases by an order of magnitude on a radial scale of 0.015 located around $r=0.62$, in the vicinity of the horizontal boundaries. Similar effects are observed on the "odd" flow spectrum distributions, for the same velocity component. This might characterize specific non-linear contributions which remain to be traced back.

At last, an exploration of the nature (traveling or standing) of the leading temporal waves is made for the "even" contribution to the flow. As appears clearly in Figure 3, the flow sign is reversed through propagative waves rather than standing ones. How to characterize traveling waves in such a small cavity, whose horizontal extension is not very different from the rolls size?

Let us recall that the following analytical description

$$
\Re\left\{\left[A_{-}(r) \exp (-i k r)+A_{+}(r) \exp (i \hbar r)\right] \exp (i \omega t)\right\}
$$

has been proposed in [4] and observed in [10] for the transient traveling waves occurring in extended cavities near the Hopf bifurcation. In this expression, $k$ is associated with the roll size and the functions $A_{-}$and $A_{+}$are slowly varying on the roll scale. In the more general case where $A_{-}$and $A_{+}$are complex, both the imaginary $\Im(r)$ and real $\Re(r)$ parts of the amplitude of $e^{2 \omega t}$ should exhibit zeros (and extrema), with two consecutive zeros (or extrema) separated, on the $r$-axis, by approximately the same distance $D=\pi / k$. Could such a behavior 


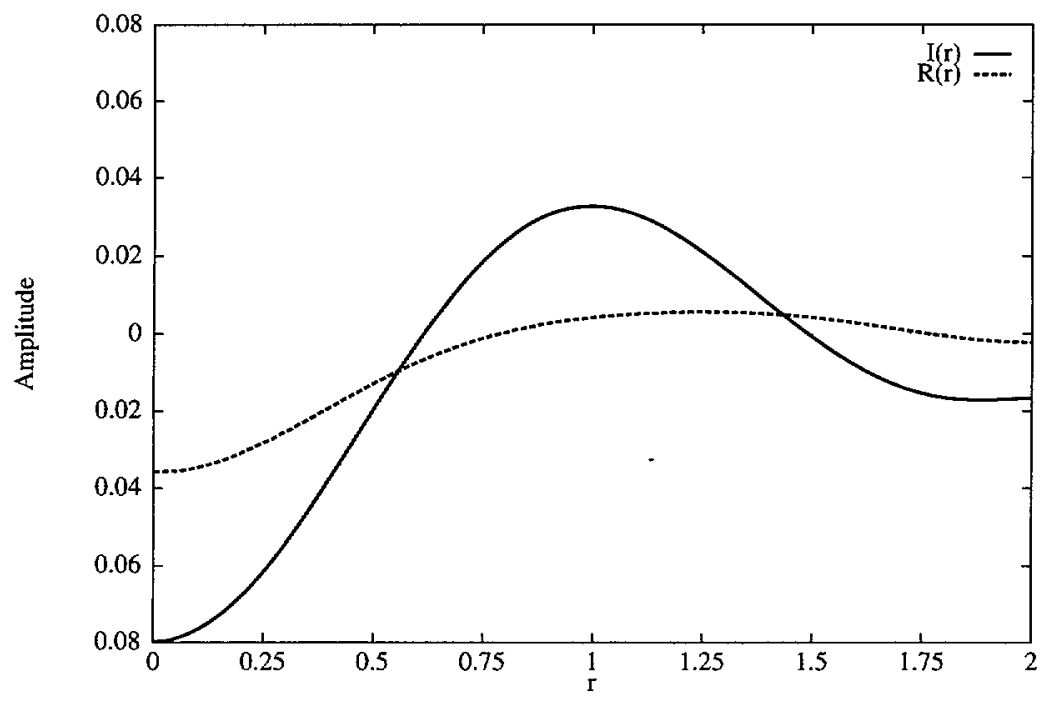

Fig. 7. - First temporal mode complex amplitude for the departure of the temperature at $z=0$ : real $\Re(r)$ and imaginary $\Im(r)$ parts.

be present in our steady oscillatory confined convective states? The first (positive frequency, say) temporal mode complex amplitude is considered now, at the mid height $z=0$ position, as a function of the radial distance $r$. Figure 7 presents its real $\Re(r)$ and imaginary $\Im(r)$ parts for the temperature field, similar behaviors being observed for the other fields. The aforementioned properties are effectively observed in the internal part of the $r$-axis, excluding. from this discussion, the modulation due to the boundary conditions.

\section{Conclusion}

We have presented numerical results (from a pseudo-spectral solver of the Oberbeck-Boussinesq) on oscillatory convection of a binary mixture in small cavities (axisymmetrical cylinder or Cartesian). They point out an abrupt transition to the stationary regime. A small radial scale is observed, near the horizontal boundaries, where a high order harmonic of the leading frequency dominates. At last, we show that standing waves are not the preferred solutions in this small cavities, but rather unbalanced and phase shifted counterpropagating traveling waves.

\section{References}

[1] Barten W., Lucke M., Kamps M. and Schmitz R., Convection in binary fluid mixture. I. Extended traveling-wave and stationary states, Phys. Rev. E 51 (1995) 5636-5661.

[2] Batoul A., Khallouf $H$. et Labrosse G., Une méthode de résolution directe (pseudospectrale) du problème de Stokes 2D/3D instationnaire. Application à la cavité entraînée carrée, C.R. Acad. Sci. Paris, t.319, Série II (1994) pp. 1455-1461. 
[3] Canuto C., Hussaini M.Y., Quarteroni A. and Zang T.A., Spectral Methods in Fluid Dynamics (Springer-Verlag, NY, 1988).

[4] Cross M.C., Traveling and standing waves in binary-fluid mixture in finite geometries, Phys. Rev. Lett. 57 (1986) 2935-2938.

[5] Haldenwang P. and Labrosse G., 2-D and 3-D spectral Chebyshev solutions for free convection at high Rayleigh number, Proc. 6th Int. Symposium on finite element methods in flow problems (1986) pp. 261-266.

[6] Heinrichs R., Ahlers G. and Cannell D.S., Traveling waves and spatial variation in the convection of a binary mixture, Phys. Rev. A 35 (1987) 2761-2764.

[7] Mercader I., Net M. and Knobloch E., Binary fluid convection in a cylinder, Phys. Rev. E 51 (1994) 339-351.

[8] Ouadi M., Étude du point de codimension deux pour le problème de Rayleigh Bénard en double diffusion, Thèse de l'Université de Nice - Sophia Antipolis (France, 30 Juin 1992)

[9] Platten J.K. and Legros J.C., Convection in Liquids (Springer-Verlag, 1984).

[10] Surko C.M. and Kolodner P., Oscillatory traveling waves convection in finite container, Phys. Rev. Letters 58 (1987) 2055-2058.

[11] Surko C.M., Kolodner P., Passner A. and Walden R.W., Binary fluid convection in a cylinder, Physica 23D (1986) 220-351. 